\title{
SEXUALIDADE NA ADOLESCÊNCIA: MITOS E TABUS
}

\author{
SEXUALITY IN ADOLESCENCE: MYTHS AND TABOOS
}

\section{SEXUALIDAD EN LA ADOLESCENCIA: MITOS Y TABÚES}

\author{
Christine Baccarat de Godoy Martins* \\ Fabiana Maria de Almeida ${ }^{* *}$ \\ Lidiane Cristina AlenCASTRO ${ }^{* * *}$ \\ Karla Fonseca de Matos ${ }^{* * * *}$ \\ Solange Pires Salomé de SouZa ${ }^{* * * *}$
}

\begin{abstract}
RESUMO
A adolescência é uma fase da vida humana em que a sexualidade se insere como uma redescoberta, trazendo certa vulnerabilidade, principalmente decorrente de mitos e tabus. Este trabalho teve como objetivo identificar alguns tabus e mitos relativos à sexualidade, vivenciados pelos adolescentes de Cuiabá-MT. Pesquisa quantitativa, cuja população de estudo foi composta por estudantes do primeiro ano do ensino médio do ensino público de Cuiabá. Os dados foram coletados por instrumento fechado e processados eletronicamente, com análises simples e bivariadas $(\mathrm{p}<0,05)$. Os adolescentes: acreditam que bebida alcoólica e drogas aumentam o desejo sexual; não relacionam a possibilidade de gravidez com a prática de sexo interfemural sem proteção; acreditam que não há riscos de gravidez durante o período menstrual; os sem experiência sexual não souberam assinalar o que influencia no prazer da relação sexual; entre os sexualmente ativos houve a presença de respostas como "quanto maior o pênis do garoto maior o prazer das meninas" e "o menino sempre terá mais prazer do que a menina"; para acontecer a relação sexual é preciso amar o parceiro (para as meninas) e apenas sentir atração (para os meninos); para os meninos há preferência em se casar com alguém virgem. As diferenças de comportamento entre sexos indicam tabus arraigados no berço cultural da sociedade, em que a educação sexual, de caráter repressor, contribuiu para que os papéis e valores sexuais de homens e mulheres se mantenham rígidos e conservadores ainda hoje, embora as novas gerações comecem a imprimir novos comportamentos. Torna-se urgente garantir ao adolescente orientação sobre o desenvolvimento de sua sexualidade, a fim de prevenir comportamentos de risco decorrentes dos tabus e mitos.
\end{abstract}

Palavras chave: Tabu, sexualidade, adolescente, saúde do adolescente.

\footnotetext{
ABSTRACT

Adolescence is a phase of human life in which sexuality appears as a rediscovery, causing certain vulnerability mainly due to myths and taboos. This study aimed to identify some taboos and myths regarding sexuality, experienced by adolescents in Cuiabá-MT. Quantitative research, whose study population was composed by

*Enfermeira, docente da Universidade Federal do Mato Grosso (UFMT), Departamento de Enfermagem, UFMT - Cuiabá, Brasil.Email: leocris2001@terra.com.br

${ }^{* *}$ Acadêmica de Enfermagem, bolsista PIBIC - UFMT - Cuiabá, Brasil. Email: fabiana_filippin@yahoo.com.br

*** Acadêmica de Enfermagem, bolsista PIBIC - UFMT - Cuiabá, Brasil. Email: lidi_alencastro@hotmail.com

${ }^{* * * *}$ Enfermeira, mestranda do Programa de Mestrado em Enfermagem da Faculdade de Enfermagem da UFMT. Bolsista CAPES. UFMT - Cuiabá, Brasil. Email: karla.matos88@gmail.com

${ }^{* * * * *}$ Enfermeira, docente da Universidade Federal do Mato Grosso (UFMT), Departamento de Enfermagem, UFMT Cuiabá, Brasil. Email: solps2@gmail.com
} 
students from the first year of Cuiabá public high school education. Data were collected by a closed answer instrument and electronically processed, with simple and bivariate analysis $(\mathrm{p}<0.05)$. Adolescents believe that alcohol and drugs increase sexual desire, do not relate the possibility of pregnancy to the practice of intercrural sex without protection, they believe there is no risk of pregnancy during the menstrual period; those without sexual experience could not tell what influences the pleasure of sexual intercourse; among those sexually active there have been responses like "the bigger the boy's penis the greater the pleasure of the girls" and "a boy will always feel more pleasure than girls"; for a sexual relation to happen one must love the partner (for girls) and just feel attraction (for boys); boys prefer to marry a virgin. The gender differences in behavior indicate taboos rooted in the cultural cradle of society, in which sexual education, repressive in nature, contributes to maintain men and women roles and sexual values firmly set even today, although the new generations have started to introduce new behaviors. It becomes urgent to guarantee the adolescent orientation on the development of their sexuality, in order to prevent risk behaviors resulting from the taboos and myths.

Key words: Taboo, sexuality, adolescent, adolescent health.

\section{RESUMEN}

La adolescencia es una fase de la vida humana en que la sexualidad se manifiesta como un redescubrimiento, provocando cierta vulnerabilidad, resultante principalmente de mitos y tabúes. Este trabajo tuvo como objetivo identificar algunos tabúes y mitos en relación con la sexualidad, vivenciados por los adolescentes de CuiabáMT. Investigación cuantitativa, cuya población de estudio estuvo integrada por estudiantes de primer año de enseñanza secundaria pública de Cuiabá. Los datos fueron recogidos por instrumentos de respuesta cerrada y procesados electrónicamente, por análisis simples y bivariadas $(\mathrm{p}<0,05)$. Los adolescentes creen que bebida alcohólica y drogas aumentan el deseo sexual; no relacionan la posibilidad de embarazo con la práctica de sexo interfemural sin protección; creen que no hay riesgos de embarazo durante el período menstrual; los que no tuvieron experiencia sexual no supieron señalar qué causa placer en la relación sexual; entre los sexualmente activos se dieron respuestas como "cuanto mayor el pene del muchacho mayor el placer de las chicas" y "el muchacho siempre tendrá más placer que las chicas"; para que haya relación sexual es preciso amar al compañero (para las chicas) y apenas sentir atracción (para los muchachos); para los muchachos hay preferencia en casarse con alguien virgen. Las diferencias de comportamiento entre sexos indican tabúes arraigados en la cuna cultural de la sociedad, en que la educación sexual, de carácter represor, contribuye para que los papeles y valores sexuales de hombres y mujeres se mantengan rígidos y conservadores aún hoy, aunque las nuevas generaciones comiencen a expresar nuevos comportamientos. Se vuelve urgente garantizar al adolescente orientación sobre el desarrollo de su sexualidad, a fin de prevenir comportamientos de riesgo resultantes de los tabúes y mitos.

Palabras clave: Tabú, sexualidad, adolescente, salud del adolescente.

Fecha recepción: 20/10/11 Fecha aceptación: 31/08/12

\section{INTRODUÇÃO}

A adolescência é uma fase da vida humana caracterizada por um conjunto de transformações de dimensões biológicas, psíquicas e sociais (1-3). É nesta fase que a sexualidade se insere como uma redescoberta de algo intrínseco do ser humano, construído ao lon- go da sua trajetória pessoal, sendo que seu exercício vai além das funções reprodutivas, estendendo-se até o processo de socialização do adolescente (4).

Sabe-se que este processo de amadurecimento traz consigo certa vulnerabilidade, principalmente decorrente de mitos e tabus ligados à sua sexualidade (5), pois os falsos conceitos e a compreensão distorcida da se- 
xualidade podem impedir o adolescente de vivê-la plenamente sem riscos (6).

Considerando que os mitos e tabus reforçam o padrão sexual e a cultura de uma determinada população e que podem contribuir para o desenvolvimento de problemas sexuais (2), torna-se essencial desmistificar conceitos equivocados e orientar os adolescentes para que exerçam sua sexualidade com segurança, tranquilidade e plenitude, contribuindo, assim, para uma prática sexual saudável $(3,4,7)$.

Autores destacam que a influência da cultura patriarcal, a religião, a escola, bem como as questões políticas e econômicas, influenciam fortemente a formação da sexualidade, em que predominam a falta de diálogo e a educação autoritária $(4,6)$, o que contribui para a existência de mitos e tabus, colocando o adolescente como importante grupo de risco. Este cenário, aliado à dificuldade das escolas em abordar o tema, demandam dos profissionais de saúde ações que possam promover a orientação sexual para este grupo (1-3).

Neste contexto, destaca-se o papel da enfermagem como membro da equipe de saúde, à qual cabe a atenção ao adolescente, inserindo a sexualidade como um elemento importante (6). Atualmente, a enfermagem necessita desenvolver tecnologias de ensino capazes de abordar a sexualidade com adolescentes de forma positiva a fim de promover a saúde sexual e reprodutiva, num diálogo franco e aberto. Seja na atenção básica, terciária, na escola ou comunidades, a equipe de saúde, principalmente a enfermagem, desempenha o importante compromisso de educador em saúde, colaborando na formação dos jovens na atualidade (4). Para tanto, é preciso que os profissionais busquem conhecer os mitos e os tabus que cercam os adolescentes no que se refere à sexualidade para que possam abordá-la de forma mais efetiva.

Diante deste contexto, este artigo objetivou identificar alguns tabus e mitos relativos à sexualidade, vivenciados pelos adolescentes matriculados no primeiro ano do ensino médio, na rede estadual de ensino do município de Cuiabá-MT.

\section{METODOLOGIA}

Trata-se de pesquisa quantitativa, realizada em 5 (cinco) escolas estaduais do município de Cuiabá - Mato Grosso, no ano de 2009. As escolas foram sorteadas aleatoriamente junto com a Secretaria de Estado de Educação de Mato Grosso (SEDUC) - amostragem probabilística aleatória simples, totalizando 22 turmas do período matutino. A população de estudo foi composta seguindo os seguintes critérios de inclusão: alunos do $1^{\circ}$ ano de ensino médio, na idade de 11 a 19 anos, período considerado pela Organização Mundial da Saúde como adolescência, além de querer participar do estudo assinando o Termo de Consentimento Livre e Esclarecido. A autorização dos pais ou responsável foi providenciada antecipadamente pela direção das escolas envolvidas.

Foram adotados como critérios de exclusão: os alunos fora da faixa etária de interesse (abaixo de 11 anos ou acima de 19 anos); os que não concordassem em participar do estudo ou que, ainda, não tivessem obtido autorização dos pais para a participação na pesquisa. Entretanto, não houve nenhum caso de exclusão.

Participaram do estudo 499 adolescentes. A coleta dos dados se deu por meio de questionário fechado, com 11 questões de múltipla escolha, aplicado em sala de aula, cujas perguntas foram: sexo; atividade sexual (ativo ou não); o que é preciso para uma relação sexual acontecer; o que influencia o prazer durante a relação sexual; se prefere casar-se com alguém virgem ou não; se bebida alcóolica e outras drogas aumentam o desejo sexual; se relação interfemural sem penetração pode engravidar; se relação sexual durante 
menstruação pode engravidar; qual o período mais propício para a gravidez acontecer; se teve informações prévias sobre sexualidade; qual foi a fonte destas informações; se as mesmas foram satisfatórias ou não. Em cada escola, agendou-se previamente com a direção da mesma o dia de aplicação do instrumento, em todas as turmas do primeiro ano do ensino médio ao mesmo tempo a fim de evitar troca de informações entre adolescentes de turmas diferentes, caso o dia de coleta de dados fosse diferente para cada turma. $\mathrm{O}$ questionário era composto por questões fechadas, de múltipla escolha. Para assegurar o sigilo e a não identificação, foram depositadas urnas nas salas de aula para que, dessa forma, o adolescente depositasse diretamente na urna seu questionário respondido.

Toda a coleta foi realizada por uma equipe de acadêmicos de enfermagem da Universidade Federal de Mato Grosso, previamente treinados e supervisionados pela pesquisadora. O instrumento de coleta de dados foi testado previamente em uma escola que não faria parte do estudo, permitindo o ajuste do mesmo.

Os dados foram processados pelo software Epi-Info versão 3.5.0 e foram estudadas as seguintes variáveis: se a bebida alcoólica ou outras drogas aumentam o desejo sexual; se há possibilidade de gravidez mesmo sem penetração; se há possibilidade de gravidez durante o período menstrual; qual o período do mês em que a garota pode engravidar; se tiveram informação sobre ejaculação ou menstruação antes destes eventos ocorrerem; se sim qual a fonte de informação e se estas foram satisfatórias; o que é preciso para sentir prazer na relação sexual; o que é preciso para acontecer a relação sexual; a preferência ou não por casar com alguém virgem. Todas as variáveis foram analisadas segundo o sexo (análise bivariada, utilizando-se o teste de qui-quadrado), em frequências absoluta (n) e relativa (\%). Houve, ainda, estratificação segundo a atividade sexual (sexualmente ativos ou não) para algumas variáveis (o que influencia o prazer durante a relação sexual e o que é preciso para uma relação sexual acontecer). Considerou-se o valor de p (probabilidade) $<0,05$ para diferenças estatisticamente significantes.

A presente pesquisa foi autorizada pela Secretária de Estado de Educação, pela diretoria das escolas e pelo Comitê de Ética em Pesquisa do HUJM, conforme protocolo número 613/CEP-HUJM/09, estando de acordo com a Resolução CNS 196/96.

\section{RESULTADOS}

Os resultados revelaram que 46,3\% dos meninos e $77,7 \%$ das meninas acreditam que o uso de bebida alcoólica e outras drogas aumentam o desejo sexual (Tabela 1). Considerando que a análise destes resultados teve o valor de $\mathrm{p}=0,0243$ segundo o sexo, podemos afirmar que as diferenças nas respostas entre meninos e meninas são estatisticamente significativas, ou seja, entre as meninas a crença e que a bebida alcóolica e outras drogas aumentam o desejo sexual é muito maior do que entre os meninos.

Quando indagados sobre a possibilidade de gravidez na relação interfemural (popular "sexo entre coxas"), observou-se que 50,5\% dos meninos e $21,1 \%$ das meninas acreditam que esta prática sexual não traz riscos de engravidar (Tabela 1). O valor de $\mathrm{p}=0,0000$ aponta para diferença estatisticamente significativa entre os sexos, ou seja, a crença de que a relação sexual interfemural não traz riscos de engravidar se modifica conforme o sexo, sendo que os meninos afirmam isto em maior proporção em relação às meninas.

A maioria dos adolescentes, tanto meninos $(45,3 \%)$ quanto meninas $(60,2 \%)$, acreditam que a relação sexual sem preservativo durante a menstruação pode acarretar em gravidez (Tabela 1). Considerando o valor de $\mathrm{p}=0,0011$, há diferenças estatisticamente significativas nas respostas conforme o sexo, 
Tabela 1. Distribuição dos adolescentes segundo o sexo e tabus relativos à sexualidade. Cuiabá, 2010.

\begin{tabular}{|c|c|c|c|c|c|c|}
\hline \multirow{3}{*}{$\begin{array}{l}\text { O uso de bebida alcoólica e outras drogas } \\
\text { aumentam o desejo sexual } \\
\qquad \begin{array}{l}p=0243\end{array}\end{array}$} & \multicolumn{4}{|c|}{ Sexo } & \multirow{2}{*}{\multicolumn{2}{|c|}{ Total }} \\
\hline & \multicolumn{2}{|c|}{ Masculino } & \multicolumn{2}{|c|}{ Feminino } & & \\
\hline & No & $\%$ & No & $\%$ & No & $\%$ \\
\hline Sim & 88 & 46,3 & 240 & 77,7 & 328 & 65,7 \\
\hline Não & 49 & 25,8 & 68 & 22,0 & 117 & 23,5 \\
\hline Não sei & 53 & 27,9 & 1 & 0,3 & 54 & 10,8 \\
\hline TOTAL & 190 & 100,0 & 3091 & 100,0 & 499 & 100,0 \\
\hline \multirow{3}{*}{$\begin{array}{l}\text { Ter relação sexual interfemural ("entre coxas") sem proteção } \\
\text { pode engravidar, mesmo sem penetração vaginal } \\
\text { p=0,0000 }\end{array}$} & \multicolumn{4}{|c|}{ Sexo } & \multirow{2}{*}{\multicolumn{2}{|c|}{ Total }} \\
\hline & \multicolumn{2}{|c|}{ Masculino } & \multicolumn{2}{|c|}{ Feminino } & & \\
\hline & No & $\%$ & No & $\%$ & No & $\%$ \\
\hline Sim & 51 & 26,8 & 168 & 54,4 & 219 & 43,9 \\
\hline Não & 96 & 50,5 & 65 & 21,0 & 161 & 32,3 \\
\hline Não sei & 43 & 22,7 & 76 & 24,6 & 119 & 23,8 \\
\hline TOTAL & 190 & 100,0 & 3091 & 100,0 & 499 & 100,0 \\
\hline \multirow{3}{*}{$\begin{array}{l}\text { Ter relação sexual sem preservativo durante a menstruação } \\
\text { pode acarretar em gravidez } \\
p=0,0011\end{array}$} & \multicolumn{4}{|c|}{ Sexo } & \multirow{2}{*}{\multicolumn{2}{|c|}{ Total }} \\
\hline & \multicolumn{2}{|c|}{ Masculino } & \multicolumn{2}{|c|}{ Feminino } & & \\
\hline & No & $\%$ & No & $\%$ & No & $\%$ \\
\hline Sim & 86 & 45,3 & 186 & 60,2 & 272 & 54,5 \\
\hline Não & 44 & 23,2 & 67 & 21,7 & 111 & 22,3 \\
\hline Não sei & 60 & 31,5 & 56 & 18,1 & 116 & 23,2 \\
\hline TOTAL & 190 & 100,0 & 3091 & 100,0 & 499 & 100,0 \\
\hline
\end{tabular}

ou seja, as meninas acreditam no risco de engravidar durante a menstruação em maior proporção do que os meninos.

Diferentemente da pergunta anterior, que indaga sobre a possibilidade de engravidar durante a menstruação (tendo relação sexual sem preservativo), a pergunta a seguir inda- ga sobre o período mais propício para se engravidar. Quando perguntados a respeito do período mais propício para que uma garota possa engravidar, 46,3\% dos adolescentes do sexo masculino e $77,7 \%$ do sexo feminino se referiram ao período fértil (Tabela 2 ). Considerando-se o valor de $p=0,0000$, observa-se

Tabela 2. Distribuição dos adolescentes segundo o sexo e o conhecimento sobre qual o período do ciclo menstrual em que pode ocorrer uma gravidez. Cuiabá, 2010.

\begin{tabular}{|c|c|c|c|c|c|c|}
\hline \multirow{3}{*}{ Período em que a garota pode engravidar } & \multicolumn{4}{|c|}{ Sexo } & \multirow{2}{*}{\multicolumn{2}{|c|}{ Total }} \\
\hline & \multicolumn{2}{|c|}{ Masculino } & \multicolumn{2}{|c|}{ Feminino } & & \\
\hline & No & $\%$ & No & $\%$ & No & $\%$ \\
\hline Período fértil & 88 & 46,3 & 240 & 77,7 & 328 & 65,8 \\
\hline Fora do período menstrual & 34 & 17,9 & 17 & 5,5 & 51 & 10,2 \\
\hline Início da menstruação & 15 & 7,9 & 5 & 1,6 & 20 & 4,0 \\
\hline Durante a menstruação & 9 & 4,7 & 6 & 1,9 & 15 & 3,0 \\
\hline Final da menstruação & 6 & 3,2 & 12 & 3,9 & 18 & 3,6 \\
\hline Não sei & 32 & 16,8 & 23 & 7,5 & 55 & 11,0 \\
\hline Em branco & 6 & 3,2 & 4 & 1,3 & 10 & 2,0 \\
\hline Respostas nulas & - & - & 2 & 0,6 & 2 & 0,4 \\
\hline TOTAL & 190 & 100,0 & 309 & 100,0 & 499 & 100,0 \\
\hline
\end{tabular}


que as meninas revelam maior conhecimento quanto ao período mais propicio para engravidar do que os meninos.

Distribuindo-se os adolescentes segundo o sexo e a presença de informações prévias sobre menstruação ou ejaculação, a maioria $(70,7 \%$ das meninas e $42,6 \%$ dos meninos) refere que teve acesso a informações, enquanto $23,7 \%$ dos meninos e $16,0 \%$ das meninas não receberam. Observou-se um número maior no sexo masculino $(29,5 \%)$ que não soube responder em relação ao sexo feminino $(11,4 \%)$, deixaram em branco $4,2 \%$ dos meninos e $2,0 \%$ das meninas. Considerando-se o valor de $\mathrm{p}(0,0000)$, nota-se que as meninas tiveram informações prévias sobre menstruação ou ejaculação em maior proporção em relação aos meninos.

Segundo a Tabela 3, as meninas tiveram a mãe como a fonte mais consultada no que se refere às informações prévias sobre menstruação $(52,4 \%)$, diferentemente dos meninos em relação à ejaculação, cuja fonte de informação foi as amigas $(23,7 \%)$, professores $(9,5 \%)$, livros e revistas $(7,9 \%)$ e outros $(10,5 \%)$. O valor de $\mathrm{p}=0,0000$ aponta para diferenças estatisticamente significativas entre os sexos quando se trata de informações sobre sexualidade, ou seja, a fonte de informação de modifica conforme o sexo do adolescente.

Tabela 3. Distribuição dos adolescentes segundo o sexo e a fonte da informação recebida sobre menstruação ou ejaculação. Cuiabá, 2010.

\begin{tabular}{|c|c|c|c|c|c|c|}
\hline \multirow{3}{*}{$\begin{array}{l}\text { Fonte da informação sobre } \\
\text { menstruação ou ejaculação }\end{array}$} & \multicolumn{4}{|c|}{ Sexo } & \multirow{2}{*}{\multicolumn{2}{|c|}{ Total }} \\
\hline & \multicolumn{2}{|c|}{ Masculino } & \multicolumn{2}{|c|}{ Feminino } & & \\
\hline & No & $\%$ & No & $\%$ & No & $\%$ \\
\hline Amiga & 45 & 23,7 & 1 & 0,3 & 46 & 9,2 \\
\hline Professor(a) & 18 & 9,5 & 18 & 5,8 & 36 & 7,2 \\
\hline Livros/ Revistas & 15 & 7,9 & 9 & 2,9 & 24 & 4,8 \\
\hline Pai & 14 & 7,4 & - & - & 14 & 2,8 \\
\hline Mãe & 13 & 6,8 & 162 & 52,4 & 175 & 35,1 \\
\hline Amigo & 8 & 4,2 & 19 & 6,1 & 27 & 5,4 \\
\hline Parente & 5 & 2,6 & 11 & 3,6 & 16 & 3,2 \\
\hline Irmão/ irmã & 3 & 1,6 & 8 & 2,6 & 11 & 2,2 \\
\hline Outros & 20 & 10,5 & 10 & 3,2 & 30 & 6 \\
\hline Em branco & 26 & 13,8 & 12 & 3,9 & 38 & 7,6 \\
\hline Mais de uma fonte & 21 & 11,1 & 57 & 18,5 & 78 & 15,7 \\
\hline Respostas nulas & 2 & 1 & 2 & 0,7 & 4 & 0,8 \\
\hline TOTAL & 190 & 100,0 & 309 & 100,0 & 499 & 100,0 \\
\hline
\end{tabular}

Entre os adolescentes que receberam informações prévias sobre ejaculação ou menstruação, a maioria $(63,7 \%$ do sexo masculino e $82,4 \%$ do sexo feminino) considerou as informações satisfatórias. Enquanto $12,1 \%$ dos meninos e $4,6 \%$ das meninas não responderam. De acordo com o valor de $\mathrm{p}=0,0000$, as meninas consideram as infor- mações satisfatórias em maior proporção do que os meninos.

A maioria das meninas considera que é necessário amar o(a) parceiro(a) $(67,1 \%)$ para acontecer uma relação sexual, outras consideram necessário sentir atração $(12,7 \%)$, apenas gostar do parceiro(a) (3,6\%), ficar com um(a) garoto(a) (1,6\%) e, ainda, 6,5\% 
assinalaram mais de uma resposta, 7,2\% não souberam opinar, $1,3 \%$ deixaram em branco. Já os meninos, $45,3 \%$ consideram que basta sentir atração para que haja uma relação sexual, seguido de amar a(o) parceira(o) $(25,8 \%)$, ficar com a(o) garota(o) $(12,6 \%)$, gostar da(o) parceira(o) $(7,4 \%)$, sendo que $6,3 \%$ escolheram mais de uma resposta, $2,1 \%$ não souberam responder e $0,5 \%$ deixaram em branco. $O$ valor de $p=0,0000$ sugere diferenças estatisticamente significativas entre os sexos, ou seja, as circunstâncias para que ocorra uma relação sexual são muito diferentes para as meninas (para as quais envolve mais sentimento) e para os meninos (para os quais basta sentir atração).

Este comportamento também se reflete no momento em que os adolescentes foram divididos segundo a atividade sexual, ou seja, sem experiência sexual e com experiência se- xual, pois os meninos declararam-se ativos sexualmente $(55,0 \%)$ em maior proporção do que as meninas $(30,6 \%)$, com um valor de $\mathrm{p}=0,0000$, ou seja, ter experiência sexual ou não se diferencia conforme o sexo do adolescente, em que os meninos a referem com maior frequência.

Para as análises das Tabelas 4 e 5, os adolescentes foram divididos segundo a atividade sexual, ou seja, sem experiência sexual $(284=56,9 \%)$ e com experiência sexual (198 $=39,7 \%)$.

Para os que se declararam sem experiência sexual, quando indagados sobre o que mais influencia o prazer na relação sexual, a resposta predominante foi "não sei", tanto para as meninas como para os meninos, resposta coerente com a falta de experiência (Tabela 4). Para os ativos sexualmente, chama a atenção as respostas, tanto dos meninos

Tabela 4. Distribuição dos adolescentes sem experiência sexual segundo o sexo e o que influencia o prazer na relação sexual. Cuiabá. 2010.

\begin{tabular}{|c|c|c|c|c|c|c|}
\hline \multirow{3}{*}{$\begin{array}{c}\text { O que influencia o prazer na relação } \\
\text { sexual segundo adolescentes sem } \\
\text { experiência sexual }\end{array}$} & \multicolumn{4}{|c|}{ Sexo } & \multirow{2}{*}{\multicolumn{2}{|c|}{ Total }} \\
\hline & \multicolumn{2}{|c|}{ Masculino } & \multicolumn{2}{|c|}{ Feminino } & & \\
\hline & No & $\%$ & No & $\%$ & No & $\%$ \\
\hline Quanto maior o pênis do garoto, maior será o prazer & 6 & 7,7 & 10 & 4,9 & 16 & $\overline{5,6}$ \\
\hline O garoto sempre terá mais prazer do que a garota & 14 & 17,9 & 15 & 7,3 & 29 & 10,2 \\
\hline O garoto sempre sabe de tudo & 1 & 1,3 & 1 & 0,5 & 2 & 0,7 \\
\hline O prazer do garoto é mais importante do que o da garota & 4 & 5,1 & 8 & 3,9 & 12 & 4,2 \\
\hline A garota deve ser experiente para sentir tesão & 3 & 3,8 & 1 & 0,5 & 4 & 1,4 \\
\hline Nenhuma das questões & 12 & 15,4 & 20 & 9,7 & 32 & 11,3 \\
\hline Não sei & 34 & 43,6 & 142 & 68,9 & 176 & 62 \\
\hline Mais de uma resposta & 3 & 3,8 & 9 & 4,4 & 12 & 4,2 \\
\hline Em branco & 1 & 1,3 & - & - & 1 & 0,4 \\
\hline TOTAL & 78 & 100,0 & 206 & 100,0 & 284 & 100,0 \\
\hline
\end{tabular}

quanto das meninas: "Quanto maior o pênis do garoto, maior será o prazer" e "O garoto sempre terá mais prazer do que a garota" (Tabela 5).

Grande parte dos meninos $(43,2 \%)$ considera importante casar-se com alguém vir- gem, enquanto $41,4 \%$ das meninas refere que a virgindade da(o) parceira(o) é indiferente (Tabela 6). Segundo o valor de $\mathrm{p}=0,7526$, as formas de valorização da virgindade se modificam conforme o sexo, mas não são diferenças estatisticamente significativas. 
Tabela 5. Distribuição dos adolescentes com experiência sexual segundo o sexo e o que influencia o prazer na relação sexual. Cuiabá. 2010.

\begin{tabular}{|c|c|c|c|c|c|c|}
\hline \multirow{3}{*}{$\begin{array}{l}\text { O que influencia o prazer na relação sexual } \\
\text { segundo adolescentes com experiência sexual }\end{array}$} & \multicolumn{4}{|c|}{ SEXO } & \multirow{2}{*}{\multicolumn{2}{|c|}{ Total }} \\
\hline & \multicolumn{2}{|c|}{ Masculino } & \multicolumn{2}{|c|}{ Feminino } & & \\
\hline & No & $\%$ & No & $\%$ & No & $\%$ \\
\hline Quanto maior o pênis do garoto, maior será o prazer & 18 & 17,3 & 10 & 10,6 & 28 & 14,1 \\
\hline O garoto sempre terá mais prazer do que a garota & 20 & 19,2 & 12 & 12,8 & 32 & 16,2 \\
\hline O garoto sempre sabe de tudo, não precisa aprender nada sobre sexo & 1 & 1 & 2 & 2,1 & 3 & 1,5 \\
\hline O prazer do garoto é mais importante do que o da garota & 4 & 3,8 & 1 & 1 & 5 & 2,5 \\
\hline A garota deve ser experiente para sentir tesão & 8 & 7,7 & 6 & 6,4 & 14 & 7,1 \\
\hline Nenhuma das questões & 36 & 34,6 & 36 & 38,3 & 72 & 36,4 \\
\hline Não sei & 13 & 12,5 & 16 & 17 & 29 & 14,6 \\
\hline Mais de uma resposta & 4 & 3,8 & 10 & 10,6 & 14 & 7,1 \\
\hline Em branco & - & - & 1 & 1,1 & 1 & 0,5 \\
\hline TOTAL & 104 & 100 & 94 & 100 & 198 & 100,0 \\
\hline
\end{tabular}

Tabela 6. Distribuição dos adolescentes com experiência sexual segundo o sexo e questões sobre virgindade. Cuiabá. 2010.

\begin{tabular}{|c|c|c|c|c|c|c|}
\hline \multirow{3}{*}{ Quanto à virgindade do(a) parceiro(a) } & \multicolumn{4}{|c|}{ SEXO } & \multirow{2}{*}{\multicolumn{2}{|c|}{ Total }} \\
\hline & \multicolumn{2}{|c|}{ Masculino } & \multicolumn{2}{|c|}{ Feminino } & & \\
\hline & No & $\%$ & No & $\%$ & No & $\%$ \\
\hline Considera importante casar-se com alguém virgem & 82 & 43,2 & 121 & 39,1 & 203 & 40,7 \\
\hline A virgindade da(o) parceira(o) é indiferente & 66 & 34,7 & 128 & 41,4 & 194 & 38,9 \\
\hline Prefere casar com alguém virgem pelo aumento da AIDS & 7 & 3,7 & 12 & 3,9 & 19 & 3,8 \\
\hline $\begin{array}{l}\text { Casaria com alguém que não fosse mais virgem desde que a(o) outra(o) } \\
\text { tivesse sido sua(seu) única(o) parceira }(0)\end{array}$ & 6 & 3,1 & 11 & 3,6 & 17 & 3,4 \\
\hline Não sei & 25 & 13,2 & 34 & 11,1 & 59 & 11,8 \\
\hline Mais de uma resposta & 3 & 1,6 & 2 & 0,6 & 5 & 1,0 \\
\hline Em branco & 1 & 0,5 & 1 & 0,3 & 2 & 0,4 \\
\hline TOTAL & 190 & 100,0 & 309 & 100,0 & 499 & 100,0 \\
\hline
\end{tabular}

\section{DISCUSSÃO E CONCLUSÕES}

Ressalta-se que os resultados observados na presente investigação dizem respeito a um estudo transversal, realizado em determinado momento, cujas características podem se modificar ao longo do tempo. Entretanto, acredita-se que os resultados obtidos contribuem para a compreensão dos mitos e tabus vivenciados pelos adolescentes frente à sexualidade.

Ao detectar que os adolescentes acredi- tam que o uso de álcool e/ou outras drogas influenciam no aumento do desejo sexual, deve-se levar em consideração que a maioria dos adolescentes confunde a perda da inibição e o impulso para tomada de decisões com o aumento do desejo sexual, pois muitos autores apontam o uso de bebida alcoólica e/ ou outras drogas como um dos fatores para a iniciação sexual precoce na adolescência, fato este intimamente ligado à forma como a sociedade aceita o consumo de bebidas alcoólicas e tabaco entre os adolescentes (8-10). Na realidade, alguns autores consideram que 
o consumo de álcool e outras drogas surge como passaporte para a permissão e liberação de comportamentos reprimidos, ora por timidez, ora por serem julgados inadequados, além de servirem como forma de ascensão social no grupo no qual o adolescente se insere e, até mesmo no seguimento dos padrões de comportamento de seus familiares e amigos $(11,12)$.

É neste momento que se faz oportuno desenvolver a cultura de hábitos saudáveis, principalmente diante da facilidade de se obter os produtos alcoólicos / drogas, bem como a pressão exercida por seus pares para a experimentação e consumo.

Quanto à prática do sexo interfemural, o resultado sugere que os meninos desconhecem mais sobre o ciclo reprodutivo e as possibilidades de gravidez através desta prática sexual, associando a gravidez apenas às relações em que haja penetração vaginal. $\mathrm{Na}$ Grécia Antiga a prática do sexo ou coito interfemural era destinada à educação masculina ateniense, a partir de um relacionamento que ocorria entre os Erastes (homens) e os Eromenos (meninos com idade entre 12 e 18 anos), também chamado de paiderastia (amor a meninos) ou homoerotismo, cuja finalidade era a transmissão de conhecimentos (13). Embora esta prática seja antiga e presente na maioria das sociedades e culturas, não foram encontradas referências desta temática relacionadas à adolescência. Contudo, relatos de adolescentes afirmam que o sexo interfemural está entre as práticas sexuais mais frequentes entre eles, nos seus primeiros contatos sexuais que antecedem a penetração vaginal e/ou anal, o que suscita a necessidade de orientar quanto à possibilidade de gravidez e transmissão de doenças sexualmente transmissíveis mediante esta prática sem proteção.

Sobre a possibilidade de gravidez nas relações sexuais sem preservativo durante o período menstrual, embora a maioria dos adolescentes reconheça o risco de gravidez nesta prática, existe, ainda, uma parcela estimável que não soube responder. Neste sentido, autores (14) referem que a exposição ao risco de engravidar está associada à não transferência de seus conhecimentos teóricos para sua prática, bem como ao fato de assumirem um comportamento impulsivo no exercício de sua sexualidade, o que reforça a necessidade de orientação sobre sexualidade.

Outros aspectos levantados são que as adolescentes ao iniciarem sua vida sexual mantêm-se confiantes num "pensamento mágico" de que nada lhes acontecerá e, ainda, que estas reconhecem a vulnerabilidade ao risco, mas só admitem após experienciarem a gravidez (14).

Como consequências do sexo sem proteção, destacam-se o despreparo da mãe adolescente em cuidar do filho e gerenciar um lar, o surgimento de crises, conflitos e desestruturação decorrentes da falta de planejamento da gravidez, bem como o aumento na incidência de aborto, morbidade e mortalidade materna (15).

Quanto ao acesso a informações sobre ejaculação ou menstruação antes do evento ter ocorrido com o adolescente, estudo (16) aponta resultado semelhante, levantando que a mãe vem em primeiro lugar como fonte consultada entre o sexo feminino e os amigos entre o sexo masculino, seguido do professor. Importante salientar que não houve procura pelo pai para obtenção destas informações entre as meninas, o que pode ser explicado pelo despreparo dos mesmos como educador sexual $(6,17)$ de seus filhos, transferindo esta responsabilidade à escola ou, ainda, negligenciando estas questões com repressão ou descaso $(2,4)$.

Autores (4) consideram a dificuldade dos pais em lidar com a sexualidade de seus filhos por não terem a compreensão clara do que lhes aconteceu na passagem da adolescência para a vida adulta, seguindo um comportamento desinformado e reprimido, passado de geração em geração. Portanto, sugere-se a necessidade de que a escola e os serviços de saúde assumam juntamente com a família o 
papel do educador sexual para os adolescentes, pois "a falta de orientação sexual leva o adolescente à desinformação, e, consequentemente, ao perigo" (3).

Estudo realizado em escolas públicas de Pitangui-MG (16) revela que as fontes de conhecimento dos adolescentes sobre sexualidade, além dos pais, amigos e professores, estão também nos meios de comunicação, principalmente televisão $(36,6 \%)$ e revistas $(32,3 \%)$. Fato que merece atenção quando se questiona a qualidade e o direcionamento das informações obtidas através destes meios, uma vez que se constata uma crescente banalização, genitalização e erotismo dos conteúdos voltados ao público de menor idade divulgados pela mídia (18), informações estas vistas pelos pais como inadequadas frente aos valores e filosofia de vida das famílias (19).

Quanto ao fato dos meninos considerarem importante o tamanho do seu órgão sexual, percebe-se uma preocupação masculina com a prova da virilidade e o desempenho sexual embutidos no aspecto do corpo. Neste sentido, a literatura aponta que existem comportamentos distintos entre os sexos: os meninos se preocupam com o desenvolvimento do corpo, os músculos e manifestam interesse de que aconteça a iniciação sexual; já as meninas valorizam a estética, preocupando-se em como se vestir e se maquiar, considerando a menarca como um marco no seu desenvolvimento corporal e psicológico (20, 21). Alguns estudos ainda apontam a relação que adolescentes fazem entre tamanho do órgão sexual e percepção de prazer $(16,21)$.

Outra característica marcante entre os sexos são os valores quanto à perspectiva de acontecer uma relação sexual, para os meninos basta existir atração sexual (física), enquanto para as meninas há necessidade de envolvimento afetivo. Essa diferença de comportamento entre sexos indica tabus arraigados no berço cultural da sociedade, uma herança do modelo de família patriarcal, no qual havia uma submissão da mulher aos desejos e mandos dos seus senhores. Autores (6) referem que a própria educação sexual desde esta época no Brasil tinha um caráter repressor, o que, conseqüentemente, contribuiu para que os papéis e valores sexuais de homens e mulheres se mantenham rígidos e conservadores ainda hoje, embora as novas gerações comecem a imprimir novos comportamentos.

No que diz respeito à preferência de casar-se com alguém virgem, este resultado demonstra a forte valorização da virgindade feminina nas sociedades ocidentais, bastante marcante no Brasil, como apontou o estudo (22) ao comparar os aspectos envolvidos na iniciação sexual, nas cidades do Rio de Janeiro e Paris, o que é coerente com autores ao afirmarem que "a idéia da virgindade como tabu está relacionada com a proibição social da relação sexual da mulher antes do casamento" (23).

A partir do desenvolvimento deste estudo, bem como pela observação de outros (1, $2,24)$ torna-se urgente a necessidade de garantir ao adolescente orientação e cuidados sobre sua saúde reprodutiva e o desenvolvimento de sua sexualidade, como já previsto no Estatuto da Criança e do Adolescente (25) e na Lei Orgânica da Saúde (26), pensando na prevenção de comportamentos de risco, assim como DTS/Aids e gravidez.

Há que se considerar neste contexto, a natureza e qualidade das ações de educação em saúde, pois estudo (27), ao avaliar a importância dada à sexualidade dentro dos Serviços de Saúde Reprodutiva em nível mundial, chama a atenção para o fato de que esta temática é frequentemente confundida ou erroneamente utilizada como uma ferramenta de contenção de natalidade.

Para tanto, se fazem necessários investimentos nos serviços de saúde para que se possa modificar os indicadores negativos como gravidez precoce, adolescentes portadores de DST/HIV, casos de violência, entre outros (10).

Destaca-se que o desafio de orientar e 
garantir a educação sexual para adolescentes demanda parcerias entre saúde e educação. Para tanto, torna-se essencial preparar o profissional de saúde e de educação para o desenvolvimento destas ações, assim como aproximar os serviços e desenvolver trocas de experiências entre estes profissionais.

Esta parceria se faz importante uma vez que estudos apontam empobrecimento e mau aproveitamento dos conteúdos de educação sexual dentro das salas de aula, à medida que estes ora são apenas transmitidos como uma aula teórica e ora permanecem camuflados nas matérias escolares como biologia, tendo os espaços para discussões que fomentem a orientação sexual cada vez mais restritos e, ainda, parte dos educadores não se encontram preparados para abordar tal temática (5).

O presente estudo evidencia alguns tabus e mitos relativos à sexualidade, vivenciados pelos adolescentes, os quais se relacionam com questões de gênero e socioculturais, podendo ter como consequência a prática sexual desprotegida. Os achados despertam para o fato de que os mitos e tabus constituem uma questão em pauta que merece investimentos em pesquisa e em programas preventivos de saúde sexual e reprodutiva.

Neste sentido, destaca-se a importância de proporcionar aos adolescentes espaços para a discussão e orientação sobre sexualidade, incluindo a questão dos mitos e tabus de forma dinâmica e participativa. A realização de oficinas de educação sexual nas escolas, pelos profissionais da Educação e da Saúde, possibilita resultados positivos, uma vez que esta metodologia pode favorecer mudanças comportamentais entre os adolescentes pela troca e vivência diárias.

Outra proposta descrita por vários trabalhos $(1,2,4)$ consiste em desenvolver atividades acadêmicas e/ou projetos de extensão nos cursos de graduação da área da saúde, considerando a redução de índices como os de gravidez na adolescência após a realização de orientações sobre sexualidade (3).
Os resultados verificados podem direcionar programas de educação sexual para adolescentes, estratégias e ações de prevenção, objetivando promover a saúde sexual e reprodutiva deste grupo.

Sugerem-se, ainda, outras pesquisas de abordagem qualitativa e quantitativa, para que se possam verificar diferentes perspectivas além das identificadas no presente estudo, em diferentes populações, de diferentes condições econômicas e educacionais.

\section{AGRADECIMENTOS:}

Agradecemos à $\mathrm{CNPq}$ pela concessão de bolsa de iniciação científica e à Fundação de Amparo à Pesquisa de Mato Grosso (FAPEMAT) pela concessão de recursos segundo o Edital No 001/2009, processo 407104/2009.

\section{REFERÊNCIAS}

1. Carvalho AM, Rodrigues CS, Medrado KS. Oficinas em sexualidade humana com adolescentes. Psicol Estud. 2005; 10(3): 377-84.

2. Soares SM, Amaral MA, Silva LB, Silva PAB. Oficinas sobre sexualidade na adolescência: revelando vozes, desvelando olhares de estudantes do ensino médio. Esc. Anna Nery. 2008; 12(3): 485-91.

3. Ximenes Neto FRG, Dias MSA, Rocha J, Cunha ICKO. Gravidez na adolescência: motivos e percepções de adolescentes. Rev Bras Enferm. 2007; 60(3): 279-85.

4. Brêtas JRS, Silva CV. Orientação sexual para adolescentes: relato de experiência. Acta Paul Enferm. 2005; 18(3): 326-33.

5. Ayres JRC. M. Adolescence and Aids: evaluation of a preventive education experience among peers. Interface-Comun, Saude, Educ. 2003; 7 (12): 113-28.

6. Cano MAT, Ferriani MGC. Sexualidade na adolescência: um estudo bibliográfico. 
Rev Lat Am Enfermagem. 2000; 8(2): 1824.

7. Campos JADB, Zuanon ACC. Educação em saúde: aspectos relevantes apontados por adolescentes. Ciênc. odontol. bras. 2004; 7(2): 55-60.

8. Taquette SR, Vilhena MM, Paula MC. Fatores associados à iniciação sexual genital: estudo transversal com adolescentes no Rio de Janeiro. Adolesc Saude. 2004; 1(3): 17-21.

9. Cruzeiro ALS, Souza LDdeM, Silva RAda, Horta BL, Muenzer RM, Faria AD, Pinheiro RT. Iniciação sexual entre adolescentes de Pelotas. Rev bras crescimento desenvolv hum. 2008; 18(2): 116-25.

10. Bertoni N, Bastos FI, Mello MB, Makuch MY, Sousa MH, Osis MJ et al. Uso de álcool e drogas e sua influência sobre as práticas sexuais de adolescentes de Minas Gerais, Brasil. Cad Saude Publica. 2009;25(6): 1350-360.

11. Vieira DL, Ribeiro M, Romano M, Laranjeira RR. Álcool e adolescentes: estudo para implementar políticas municipais. Rev Saude Publica. 2007; 41(3): 396-403.

12. Viera PC, Aerts DRGC, Freddo SL, Bittencourt A, Monteiro L. Uso de álcool, tabaco e outras drogas por adolescentes escolares em município do Sul do Brasil. Cad Saude Publica. 2008; 24(11): 2487498.

13. Corino LCP. Homoerotismo na Grécia Antiga - homossexualidade e bisexualidade, mitos e verdades. Biblos. 2006; 19: 19-24.

14. Silva MA, Batista AA, Oliveira JP. A percepção do risco de gravidez na adolescência. [Internet]. Goiás: PUC Goiás; s.f. [citado 06 abril 2011]. Disponível em: http://www.ucg.br/ucg/institutos/nepss/ monografia/monografia_06.pdf.

15. Gurgel MGI, Alves MDS, Vieira NFC, Pinheiro PNC, Barroso GT. Gravidez na adolescência: tendência na produção científica de Enfermagem. Esc. Anna Nery. 2008; 12(4): 799-805.
16. Cançado AEL. Avaliação dos conhecimentos e conteúdos curriculares sobre sexo e sexualidade de adolescentes nas escolas públicas de Pitangui-MG [Dissertação Mestrado]. [Faculdade de Medicina, Universidade Federal de Minas Gerais]: Belo Horizonte; 2007. 162 p.

17. Borges ALV, Latorre MdoR, Schor N. Fatores associados ao início da vida sexual de adolescentes matriculados em uma unidade de saúde da família da zona leste do Município de São Paulo, Brasil. Cad Saude Publica. 2007; 23(7): 1583-594.

18. Toneli MJF, Vavassori MB. Sexualidade na adolescência: um estudo sobre jovens homens. Interações. 2004; 9(18): 109-26.

19. Cano MAT, Ferriani MGC. A família frente à sexualidade dos adolescentes. Acta Paul Enferm. 2000; 13(1): 38-46.

20. Brêtas JRS, Moreno RS, Eugênio DS, Paquier DC, Vieira TF, Bruno PR. Os rituais de passagem segundo adolescentes. Acta Paul Enferm. 2008; 21(3): 404-11.

21. Damasceno VO, Vianna VRA, Vianna JM, Lacio M, Lima JRP, Novaes JS. Imagem corporal e corpo ideal. R. bras. Ci. e Mov. 2006; 14(1): 81-94.

22. Bozon M, Heilborn ML. As carícias e as palavras: Iniciação sexual no Rio de Janeiro e em Paris. Novos Estud CEBRAP. 2001; 59(2): 1-5.

23. Barreto MI, Araújo MIO, Santos CM. O olhar de alunos e alunas de uma escola municipal de Aracaju sobre a virgindade feminina e masculina. ST 53: Gênero e sexualidade na escola e na mídia. En: Fazendo Gênero 8 - Corpo, Violência e Poder [Internet]. Florianópolis; 2008 [citado 06 abril 2011]. Disponível em: www. fazendogenero8.ufsc.br/st53.html.

24. Almeida ADL, Silva CF, Cunha GS. Os conhecimentos, atitudes e comportamentos sobre sida dos adolescentes portugueses do meio urbano e não-urbano. Rev Esc Enferm USP. 2007; 41(2): 180-86. 25. Brasil. Lei 8.069, de 13 de Julho de 1990. Dispõe sobre o Estatuto da Criança e do 
Adolescente e dá outras providências. Diário Oficial da União, Brasília, DF, 13 jul. 1990.

26. Brasil. Lei 8.080, de 19 de Setembro de 1990. Dispõe sobre as condições para a promoção, proteção e recuperação da saúde, a organização e o funcionamento dos serviços correspondentes e dá outras providências. Diário Oficial da União, Brasília, DF, 19 set. 1990.

27. Dowsett GW. Algumas considerações sobre sexualidade e gênero no contexto da Aids. Questões de Saúde Reprodutiva. 2006; 1(1):39-49. 